\title{
Fabrication and Degradation of Electrospun Scaffolds from L-Tyrosine-Based Polyurethane Blends for Tissue Engineering Applications
}

\author{
Michael Spagnuolo and Lingyun Liu \\ Department of Chemical and Biomolecular Engineering, The University of Akron, Akron, OH 44325-3906, USA \\ Correspondence should be addressed to Lingyun Liu, lliu@uakron.edu
}

Received 27 October 2011; Accepted 16 November 2011

Academic Editors: B. Coasne, A. N. Obraztsov, and Y. Zhang

Copyright (C) 2012 M. Spagnuolo and L. Liu. This is an open access article distributed under the Creative Commons Attribution License, which permits unrestricted use, distribution, and reproduction in any medium, provided the original work is properly cited.

It is important to control the degradation rate of a tissue-engineered scaffold so that the scaffold will degrade in an appropriate matching rate as the tissue cells grow in. A set of potential tissue engineering scaffolds with controllable rates of degradation were fabricated from blends of two biocompatible, biodegradable L-tyrosine-based polyurethanes $\left(\mathrm{PEG}_{1000}-\mathrm{HDI}-\mathrm{DTH}\right.$ and $\mathrm{PCL} \mathrm{L}_{1250}$ HDI-DTH) using the electrospinning process. The scaffolds were characterized by mat morphology, fiber diameter, diameter distribution, pore size, and hydrolytic degradation behavior. The majority of the scaffolds, despite having radically different chemical compositions, possessed no statistical difference with pore sizes and fiber diameters. The degradation pattern observed indicated that scaffolds consisting of a greater mass percentage of PEG $_{1000}$-HDI-DTH decayed to a greater extent than those containing higher concentrations of $\mathrm{PCL}_{1250}$-HDI-DTH. The degradation rates of the electrospun scaffolds were much higher than those of the thin cast films with same compositions. These patterns were consistent through all blends. The work demonstrates one practical method of controlling the degradation of biopolymer scaffolds without significantly affecting an intended morphology.

\section{Introduction}

Biomaterials are a class of materials that interact in a desirable way with their intended living hosts. The source of such materials spans both naturally occurring and synthetic means of acquisition and each material, whether native or synthetic, presents its own set of challenges and benefits. Substances such as silk, collagen, metals, ceramics, and polymers, have all been used to solve a problem requiring a biomaterial solution. The two major concerns when choosing or designing a biomaterial for any specific application are: how the material functions in the body and how the body reacts to the presence and function of the material.

Native materials, such as collagen, are advantageous in that the living host (i.e., human) has an evolutionary means of handling and eventually disposing of the substance through hydrolytic, oxidative, and enzymatic means. Additionally, the body is able to recognize the substance as "friendly" and limit the immune response. The frequent disadvantage is that collagen by itself, for example, is often unsuited for the mechanical stresses placed on the material for many applications [1]. Polymers present an interesting alternative solution to the aforementioned problems. Synthetic polymers possess the useful property of relative ease in functional changes. Molecular number $(\mathrm{Mn})$, functional ligands, and even backbone properties can be manipulated through purely chemical means. An increase in Mn often corresponds with increased mechanical strength [2] while the proper chemical structure allows for increased biocompatibility [3]. Additionally, manipulation of the final product often requires much more mild fabrication conditions as compared to other substances such as metals and ceramics $[4,5]$.

Utilizing the appropriate biomaterial to solve a problem concerning the function of the body is one part of the expanding field of tissue engineering. A biomaterial device, fabricated in a specific manner and possibly treated after fabrication, ideally facilitates the growth or recovery of 
a desirable type of tissue in the host body upon implantation or application. One such device is referred to as a tissue engineering scaffold (TES). A TES is a porous biocompatible material that serves two functions: (1) provide necessary mechanical support and/or a protective barrier to a wounded area and (2) facilitates accelerated recovery by acting as an extracellular matrix (ECM) mimic [6]. The latter would, in theory, allow the body to bypass the time-consuming process of forming the rough collagen and fibronectin scaffold that eventually becomes the repaired extracellular matrix of the healed wound [7]. Generally, three fabrication methods have been employed to create such a structure: phase inversion, particulate leaching, and electrospinning.

Phase inversion is a process by which a solvent to the polymer and a nonsolvent to both the polymer and its solvent (often volatile) are combined together under agitation. After mixing, the environmental variables are changed such that both solvents are removed, leaving a porous polymer scaffold with thin walls separating the pores $[8,9]$. This method produces a great number of interconnected pores, but precise control is limited and pore size varies greatly. Particulate leaching, on the other hand, produces pores of uniform size, but with minimal guarantee of interconnectivity. Particulate leaching is accomplished by dissolving the polymer in a volatile organic solvent and thoroughly mixing an insoluble particulate (e.g., sodium chloride) into the solution. The solution/solid mixture is then dried and, afterwards, soaked/washed with a solvent for the solid (e.g., water) $[10,11]$. The third fabrication method is electrospinning. A polymer solution in volatile solvent is passed through a metal needle that is attached to a high voltage DC source. The stream becomes charged and a jet of quickly drying polymer solution is ejected from the needle tip. The jet passes through a linear ejection regime and then into a chaotic one where the stream is randomly tossed about before landing on a grounded stage as a nano- to microscale dry polymer fiber [12]. Electrospun fibers mimic the structures of the natural ECM morphologically and possess large surface-area-tovolume ratio and high porosity with interconnected pores, making them promising candidates as tissue engineering scaffolds.

This work sets out to fabricate tissue engineering scaffolds, using the electrospinning method, that are capable of controlled degradation in the body. The concept behind this is to allow for yet another variable to customize when attempting to design an ideal scaffold. It is important to control the degradation rate of a tissue-engineered scaffold so that the scaffold will degrade in an appropriate matching rate as the tissue cells grow in.

Two pseudo-polypeptide polyurethanes, or L-tyrosinebased polyurethanes, utilizing differing polyols and similar hard segments/chain extenders, were chosen to fabricate the scaffolds. The more hydrophilic (and thus faster degrading) polyurethane was synthesized from soft segment of poly(ethylene glycol) (PEG), a linear six-carbon diisocyanate hard segment of hexamethylene diisocyanate (HDI), and a L-tyrosine-based chain extender desaminotyrosine tyrosyl hexyl ester (DTH) [13]. This polymer is denoted as $\mathrm{PEG}_{1000^{-}}$ HDI-DTH. The second, more hydrophobic (and thus slower degrading) polyurethane was synthesized from soft segment of poly(caprolactone) (PCL), the same diisocyanate hard segment of HDI, and the same L-tyrosine-based chainextender DTH [13]. This polymer is denoted as $\mathrm{PCL}_{1250^{-}}$ HDI-DTH. The diisocyanate HDI and the amino-acid-based chain extender DTH were chosen to maintain biocompatibility of the polymers. Both polymers were synthesized via twostep condensation polymerization and characterized afterwards. The two polymers were homogeneously combined in different ratios, fabricated into fibrous scaffolds using the electrospinning process, and the final products were subjected to hydrolytic degradation testing.

\section{Materials and Methods}

2.1. Materials. Thionyl chloride, n-hexanol, tetrahydrofuran, N-(3-dimethylaminopropyl)- $\mathrm{N}^{\prime}$-ethylcarbodiimide hydrochloride (EDC-HCl), poly(ethylene glycol) $(\mathrm{Mn}=1,000)$ $\left(\mathrm{PEG}_{1000}\right)$, 1,6-hexamethylene diisocyanate (HDI), and tin(II) ethylhexanoate were purchased from Sigma-Aldrich in purities of $\geq 98 \%$. Crystalline L-tyrosine (99\%) was purchased from Acros Organics. Anhydrous diethyl ether, sodium carbonate, dichloromethane, and sodium chloride were obtained from Fisher Scientific. Anhydrous, aminefree dimethylformamide (DMF), and 3-(4-hydroxyphenyl)propionic acid were purchased from Alfa Aesar. Anhydrous magnesium sulfate was purchased from EMD Chemicals. Poly (caprolactone) diol $(\mathrm{Mn}=1,250)\left(\mathrm{PCL}_{1250}\right)$ was obtained from Polysciences. 1,1,1,3,3,3-hexafluoro-2propanol (HFIP) was purchased from Matrix Scientific. DMF was treated with drying agent $\left(\mathrm{CaH}_{2}\right)$ before use. Ltyrosine, $\mathrm{PEG}_{1000}$, and $\mathrm{PCL}_{1250}$ were dried at room temperature under vacuum for 24 hours prior to reaction. All other reagents were used as provided.

2.2. Synthesis of the Polyurethanes. The two polyurethanes, PEG $_{1000}$-HDI-DTH and PCL 1250 -HDI-DTH, were synthesized using a procedure similar to those reported in literature [13-16]. The synthesis primarily involves two steps: (1) synthesis of the chain extender DTH by a carbodiimidemediated condensation reaction [13] and (2) synthesis of the polyurethane by condensation polymerization with PEG or PCL as the macrodiol, HDI as the diisocyanate, and DTH as the chain extender. Briefly, the mixture of macrodiol and HDI ( $1: 2$ molar) in dried DMF was stirred for $3 \mathrm{~h}$ at $85^{\circ} \mathrm{C}$ to form prepolymer $\left(\mathrm{PCL}_{1250}-\mathrm{HDI}\right.$ or $\left.\mathrm{PEG}_{1000}-\mathrm{HDI}\right)$. At this stage, the literature procedure dictated a temperature of $110^{\circ} \mathrm{C}$. In order to reduce the chance of side reactions [17], a reaction temperature of $85^{\circ} \mathrm{C}$ was substituted in our work. Subsequently, the prepolymer was cooled down to room temperature and DTH ( $1: 1$ molar ratio of macrodiol : DTH) was added to the solution and reacted for $20 \mathrm{~h}$ at $80^{\circ} \mathrm{C}$ under stirring. The completed, washed polymers were dried in vacuum oven under $40^{\circ} \mathrm{C}$ for 24 hours prior to any characterization and application. Structures of $\mathrm{PCL}_{1250^{-}}$ HDI-DTH and PEG $1000-$ HDI-DTH are shown in Figure 1.

Next, the purified bulk L-tyrosine polyurethanes were characterized by means of Fourier-transform infrared 
PCL $_{1250}$-HDI-DTH:

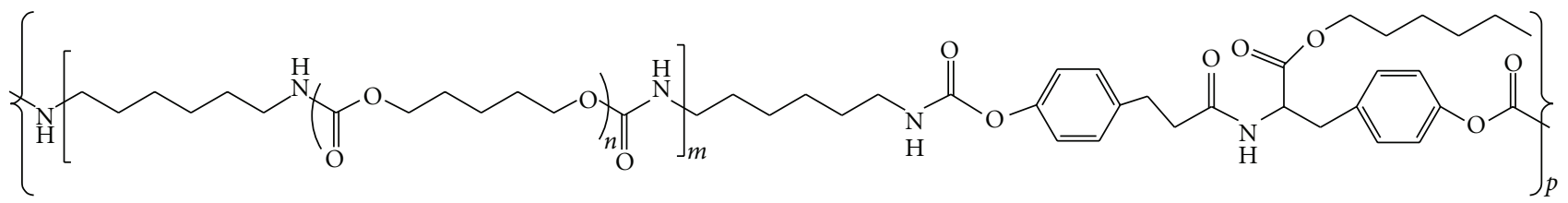

PEG $1000-H D I-D T H$

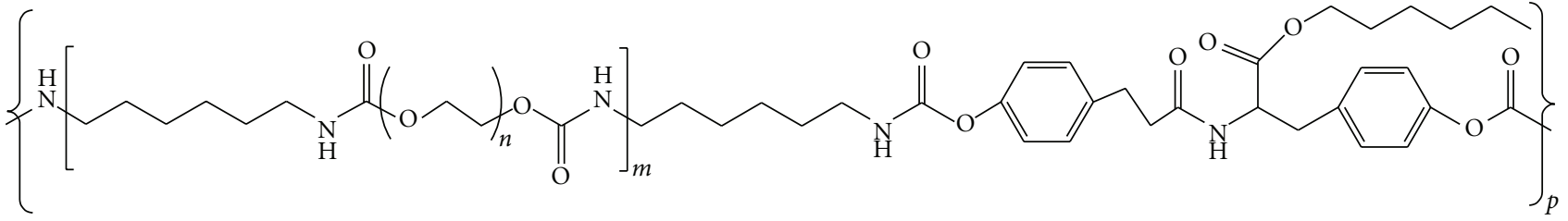

Figure 1: Chemical structure of $\mathrm{PCL}_{1250}$-HDI-DTH and $\mathrm{PEG}_{1000}-\mathrm{HDI}-\mathrm{DTH}$.

spectroscopy, thermogravimetric analysis, and differential scanning calorimetry.

\subsection{Fourier-Transform Infrared Spectroscopy (FTIR). FTIR} was used to determine the presence of specific functional groups and, in doing so, aid in elucidation of the structure of the bulk polymer. A Thermo Nicolet 380 Spectrometer was used to analyze a thin $(<1 \mathrm{~mm})$ HFIP (solvent-) cast film of both polymers. Analysis was conducted in atmosphere. Background and specimen scans consisted of 32 consecutive rescans per acquisition.

\subsection{Thermogravimetric Analysis (TGA). A TA Instruments} Thermo-Gravimetric Analyzer 2950 with platinum dishes was used for the TGA characterization. Approximately, $7 \mathrm{mg}$ of pure polyurethane sample was used per run. Samples were heated from room temperature to $600^{\circ} \mathrm{C}$ in an $\mathrm{N}_{2}$ environment at a heating rate of $20^{\circ} \mathrm{C} / \mathrm{min}$.

2.5. Differential Scanning Calorimetry (DSC). A TA Instruments Differential Scanning Calorimeter 2920 was used to analyze the pure PCL $_{1250}-\mathrm{HDI}-\mathrm{DTH}$ and $\mathrm{PEG}_{1000}-\mathrm{HDI}-\mathrm{DTH}$ samples. Approximately, $5 \mathrm{mg}$ of pure polymer was placed into aluminum pan and hermetically sealed. The DSC was run from $-100^{\circ} \mathrm{C}$ to $250^{\circ} \mathrm{C}$ using liquid $\mathrm{N}_{2}$ as the cold source and electric heating as the heat source at a heating rate of $10^{\circ} \mathrm{C} / \mathrm{min}$. Aluminum pan was used as the standard.

2.6. Blend Preparation. Five sample solutions were prepared, denoted as Blend 1 through Blend 5, in accordance to the mass ratios presented in Table 1 . Note that the Blends 1 and 5 are not truly blends, but rather pure polymers used for comparison. The blends were prepared by dry mixing the solid polymers in the appropriate ratios and then dissolving them in the common solvent HFIP.
TABLE 1: Mass ratios of polyurethanes comprising each blend and analysis of scaffold fiber diameters (mean \pm standard deviation).

\begin{tabular}{lcc}
\hline & $\begin{array}{c}\text { PEG }_{1000} \text {-HDI-DTH: } \\
\text { PCL }_{1250} \text {-HDI-DTH }\end{array}$ & Fiber diameter $(\mathrm{nm})$ \\
\hline Blend 1 & $1: 0$ & $1193.4 \pm 226.8$ \\
Blend 2 & $2: 1$ & $2205.1 \pm 940.8$ \\
Blend 3 & $1: 1$ & $1763.9 \pm 618.2$ \\
Blend 4 & $1: 2$ & $2200.7 \pm 803.0$ \\
Blend 5 & $0: 1$ & $1777.8 \pm 575.4$ \\
\hline
\end{tabular}

2.7. Electrospinning. The electrospinning apparatus (Figure 2) used consists of a syringe pump (SP101I, WPI, Sarasota, FL, USA), a high-voltage DC power supply (ES30P-5W, Gamma High Voltage Research Ormond Beach, FL), PTFE tubing (AdvantaPure, NewAge Industries, Southampton, PA), a $5 \mathrm{~mL}$ syringe (BD, Franklin Lakes, NJ, USA), with the needle tip manually blunted and deburred, and a grounded target (stage) of nonstick aluminum foil. During the electrospinning process, the polymer solution is flowed, via syringe pump, from the body of the syringe, through the PTFE tubing to the stainless steel needle. The needle is connected to the positive end of the DC power supply, effectively inducing a charge in the polymer solution. Once the solution reaches the tip, the strong electrostatic force causes a nano-to-microscale jet of solution to be ejected and travel rapidly through the air to the electrically grounded stage (collection plate). Ideally, the jet, due to high surface area, loses the majority of the often volatile solvent and a solid thread of polymer lands on the plate. These randomly deposited threads build up and eventually form a porous fiber mat which is, in this case, referred to as a tissue engineering scaffold. All five blends of the polyurethanes were fabricated into tissue engineering scaffolds in this manner. In order to reduce the number of potential variables, 


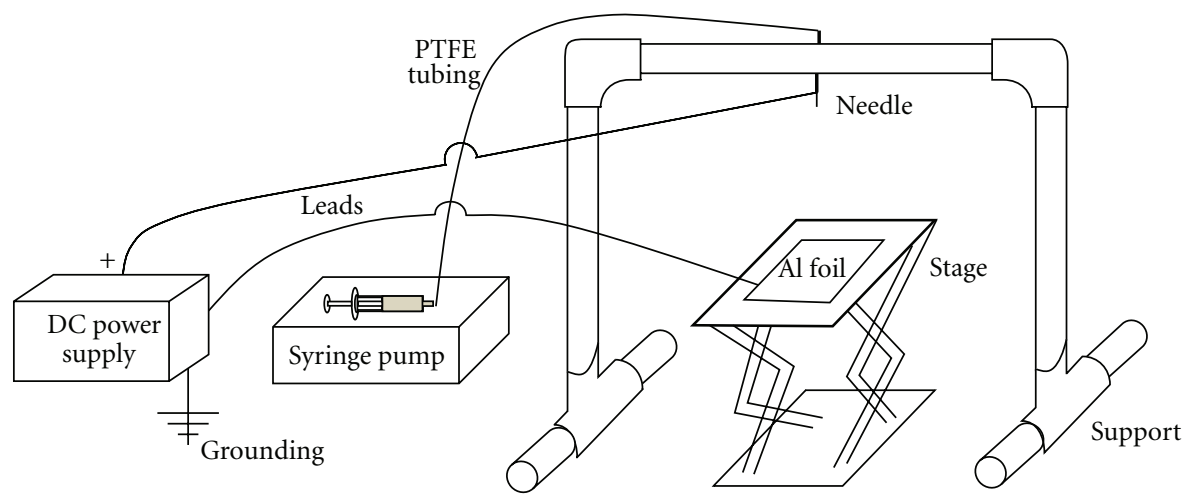

FIgURE 2: Simplified depiction of the electrospinning apparatus.

a uniform set of electrospinning parameters were employed. In all cases, a polymer solution concentration of $20 \%(\mathrm{w} / \mathrm{v})$, tip-to-collector distance of $17 \mathrm{~cm}$, voltage of $25 \mathrm{kV}$, and solution flow rate of $20 \mu \mathrm{L} / \mathrm{min}$ were used. The completed scaffolds were then morphologically characterized with the aid of scanning electron microscopy, task-appropriate measurement software, and a statistical analysis software suite.

2.8. Scanning Electron Microscopy (SEM). The electrospun scaffolds were analyzed using an FEI Quanta 200, operating under high-vacuum conditions at an accelerating voltage of $20-25 \mathrm{kV}$. All samples were coated with a thin layer of silver prior to imaging. The fibrous scaffolds were characterized by fiber diameter, fiber diameter distribution, pore size, and general topography.

2.9. Fiber Diameter. Fiber diameter was determined by analyzing the SEM micrographs using the image software ImageJ $1.43 \mathrm{u}$ and employing its scale bar calibrated measurement function. For each of the five blends, 75 diameter measurements were taken and weighed by fiber length to determine the overall mean fiber diameter of each blend.

2.10. Fiber Diameter Distribution. Fiber diameter distribution was determined using the raw unweighed fiber diameter data from the SEM analysis and subjecting it to the statistical analysis. Specifically, a histogram was created and analyzed for outliers.

2.11. Pore Size. Mean pore size was determined with the aid of the ImageJ software. Rather than simply measuring two random diameters of a pore, the entire void was outlined and the area was calculated by a scale bar-weighed measurement function of the software. This area was converted into a diameter of an area-equivalent circle in an effort to marginalize the nonuniformity effects created by the electrospinning process. Ten pores per sample were analyzed in this way to generate the mean value for each blend.

2.12. Hydrolytic Degradation. The hydrolytic degradation tests were performed in a manner similar to the method used by Sen Gupta and Lopina [13]. Briefly, $8 \mathrm{~mm}$ circular punches of each blend scaffold were vacuum-dried for 24 hours, individually weighed, placed into its own sealed vial of $10 \mathrm{~mL}$ phosphate-buffered saline (pH.4, containing $200 \mathrm{mg} / \mathrm{L} \mathrm{NaN}_{3}$ as a biocide) and stored at $37^{\circ} \mathrm{C}\left( \pm 3^{\circ} \mathrm{C}\right)$. The degraded samples were extracted at different collection points spanning over a 60 -day time period. At the time of extraction, samples (3 replicates) were removed from the incubator, gently washed with deionized water to remove latent PBS salts, vacuum-dried at $40^{\circ} \mathrm{C}$ for 48 hours and weighed. Degradation (percent mass lost, $w$ ) was calculated using the following equation:

$$
w=\frac{m_{1}-m_{2}}{m_{1}} \times 100 \%,
$$

where $m_{1}$ and $m_{2}$ are the dry mass of the sample before and after the hydrolytic degradation, respectively.

For comparison, cast thin films of each blend were also hydrolytically degraded in a similar manner. Thin film samples of each blend were cast from $10 \mathrm{wt} \%$ polymer solutions in HFIP in PTFE dishes, dried for 24 hours in atmosphere, vacuum-dried for an additional 24 hours at room temperature, punched into $8 \mathrm{~mm}$ circular discs, and immediately used for the degradation experiments.

2.13. Statistical Analysis. Analysis of variance (ANOVA) and Tukey's multiple comparison tests were performed on the data from fiber diameter, pore size, and hydrolytic degradation results, using the Minitab 15 Statistical Software suite. A confidence interval of $95 \%$ was chosen and $P<0.05$ represented the statistically significant difference.

\section{Results and Discussion}

3.1. Fourier-Transform Infrared Spectroscopy. Two polyurethanes, PCL 1250 -HDI-DTH and PEG $_{1000}-\mathrm{HDI}-\mathrm{DTH}$, were synthesized by condensation polymerization and characterized by FTIR. Figure 3 shows the stacked FTIR spectra of both polymers. The most striking feature is that, despite behaving very differently under degradation conditions as discussed later, they are chemically very similar. Given the structure of the polymers, this should not be a surprise as the only major difference occurs in the polyol soft segment. 


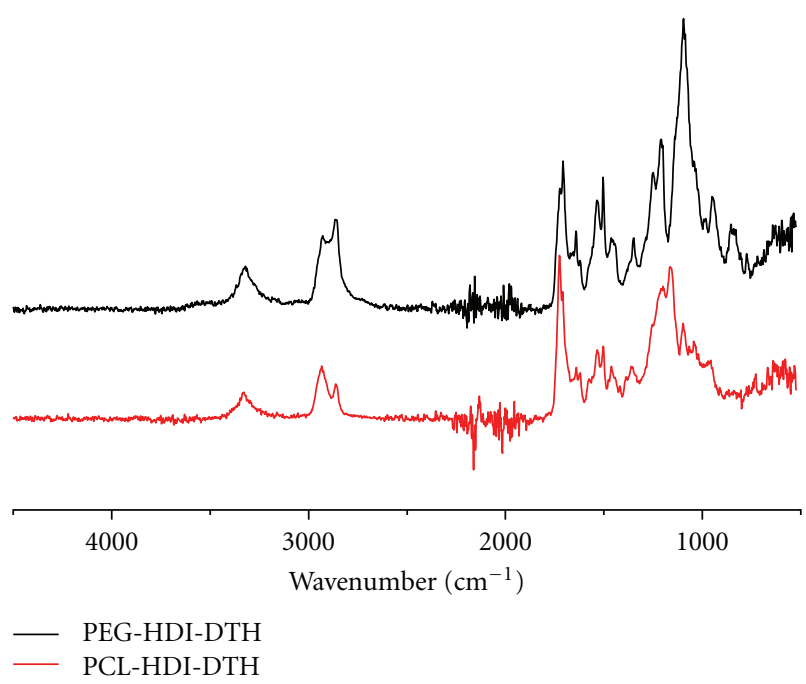

FIGURE 3: FTIR absorbance spectra of $\mathrm{PCL}_{1250}-\mathrm{HDI}-\mathrm{DTH}$ and PEG $_{1000}$-HDI-DTH.

The hard isocyanate segment and the DTH chain extender segment are identical.

The relevant peaks of $\mathrm{PCL}_{1250}$-HDI-DTH and $\mathrm{PEG}_{1000^{-}}$ HDI-DTH are briefly discussed here. The first spectrographic peak occurs around $3300 \mathrm{~cm}^{-1}$. This is very likely the hydrogen bonding stretch from $\mathrm{N}-\mathrm{H}$. It usually has a much broader base, however the sharpened peak observed here in both samples can be explained by the hindered hydrogen bonding [18]. The next two peaks occur around $2950 \mathrm{~cm}^{-1}$ and $2850 \mathrm{~cm}^{-1}$ and are easily explained as asymmetric and symmetric stretching of $\mathrm{CH}_{2}$, respectively. The peak at $1730 \mathrm{~cm}^{-1}$ represents the urethane carbonyl, which is heavily marked in the $\mathrm{PCL}_{1250}$-HDI-DTH sample as PCL contains much more carbonyl groups than PEG. The peak near $1600 \mathrm{~cm}^{-1}$ likely represents the presence of an aromatic group. Given that the only way an aromatic would be present is if the only aromatic containing compound, DTH, was successfully incorporated into the polymer, this strongly suggests that the three major components of the polymer were successfully reacted. The peak near $1200 \mathrm{~cm}^{-1}$ is an alkyl $\mathrm{C}-\mathrm{N}$ stretch. Finally, the peak near $1100 \mathrm{~cm}^{-1}$ represents the alkyl ether linkages which are much more plentiful in $\mathrm{PEG}_{1000}$-HDI-DTH as PEG contains numerous such bonds. Using these peaks as a basis, the FT-IR results support the presented molecular structure of the two L-tyrosine-based polyurethanes.

3.2. Thermogravimetric Analysis. The TGA thermograms of two pure polymers are shown in Figure 4. Results indicate a degradation temperature of approximately $300^{\circ} \mathrm{C}$ for both polyurethanes, consistent with the previous literature work [14]. A decomposition temperature near $300^{\circ} \mathrm{C}$ allows for a large thermal processing range of these materials especially considering the designed application targets the body, which rarely sees local temperatures greater than $40^{\circ} \mathrm{C}$.

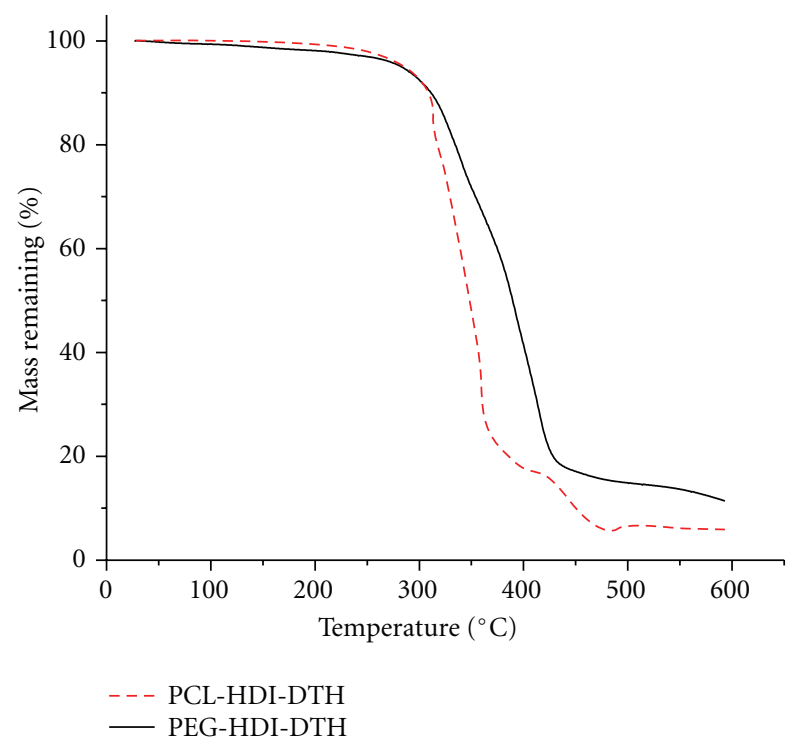

FIgure 4: TGA thermograms of $\mathrm{PCL}_{1250}$-HDI-DTH and $\mathrm{PEG}_{1000^{-}}$ HDI-DTH.

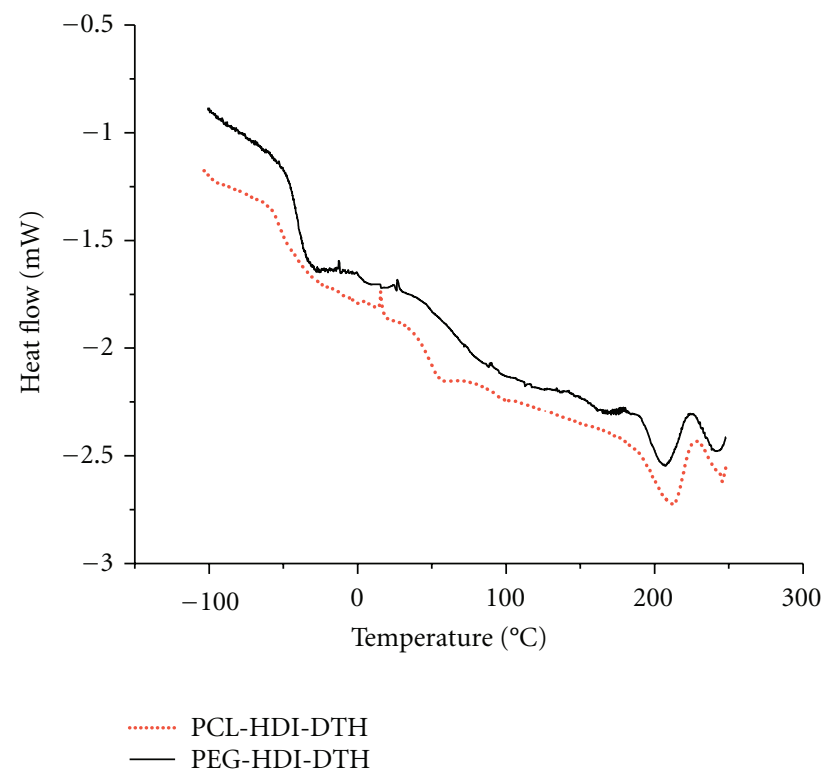

FIGURE 5: DSC thermograms of PCL 1250 -HDI-DTH and $\mathrm{PEG}_{1000}$ HDI-DTH.

3.3. Differential Scanning Calorimetry. The DSC thermograms in Figure 5 provided several interesting pieces of data, most notably the glass transition temperatures $(\mathrm{Tg})$ of the two pure polyurethanes. $\mathrm{PEG}_{1000}$-HDI-DTH experienced a $\mathrm{Tg}$ at approximately $-40^{\circ} \mathrm{C}$ while $\mathrm{PCL}_{1250}$-HDI-DTH appeared to show two transitions: one near $-34^{\circ} \mathrm{C}$ and the other at approximately $44^{\circ} \mathrm{C}$. The second $\mathrm{Tg}$ of $\mathrm{PCL}_{1250^{-}}$ HDI-DTH is very likely attributed to the hard segment (HDI and DTH). The visibility of this point would indicate a slightly amorphous hard segment. Other than the glass transitions, the polyurethanes react very similar to the large change in temperature until temperatures approach $200^{\circ} \mathrm{C}$ 
and endothermic peaks appear. These peaks likely denote the melting points of the polyurethanes. This behavior, in combination with the TGA results, suggests that both $\mathrm{PEG}_{1000}$ HDI-DTH and PCL 1250 -HDI-DTH are thermally stable.

3.4. Fibrous Scaffold Morphology. The electrospinning process was employed to fabricate fibrous tissue engineering scaffolds from blended solutions of two L-tyrosine based polyurethanes ( PCL $_{1250}$-HDI-DTH and PEG $_{1000}-$ HDI-DTH) in volatile solvent HFIP. The ratios used for each blend are shown in Table 1. All blends were spun under the same conditions and device settings to study the composition effect and reduce as many other variables as possible. Macroscopically, the scaffolds all appeared chalk-white, smooth, and elastic, resembling elastic pieces of copy paper. SEM images of all five scaffold samples are shown in Figure 6. All five blended scaffolds had major similarities and some differences.

The fibers from Blend 1 (100\% PEG $\left._{1000}-\mathrm{HDI}-\mathrm{DTH}\right)$ possessed a heavily webbed structure with significant nodal fusion (Figure 6(a)). The fibers were uniform in appearance and, upon high-resolution SEM inspection, appeared to have a relatively smooth surface. One potential cause of the webbed structure was the presence of relatively excessive solvent in the spinning solution. Although all blends were spun at the same mass/volume concentration, hydrophilic nature of the soft segment PEG may slow down the evaporation rate of solvent, causing "wet" fibers stuck and fused together. Blend 1 fibers also possessed the most uniform pore shape among five blends, very likely due to the seemingly ordered "branch and stem" fiber structure.

Unlike those from Blend 1, scaffolds of Blend 2 (67\% $\mathrm{PEG}_{1000}$-HDI-DTH, 33\% PCL 1250 -HDI-DTH) appeared as a more traditional, long, mostly unbranched fiber mat with minimal fusing and no visible nodal formation (Figure 6(b)). A small amount of secondary low-diameter fibers were observed in this sample. Fiber surface appeared visually homogenous.

Blend 3 (50\% PEG 1000 -HDI-DTH, 50\% PCL 1250 -HDIDTH) appeared as mat of long, moderately branched fibers with minimal axial node formation (Figure 6(c)). The secondary small-diameter fibers observed in Blend 2 were also present in this blend.

Blend 4 (33\% PEG 1000 -HDI-DTH, 67\% PCL 1250 -HDIDTH) possessed large primary fibers with noticeable branching (Figure 6(d)). Like previous blends, small, secondary fibers were present in this sample. Unlike previous blends, however, the fibers exhibited considerably more "looping" of the individual strands.

Blend 5 (100\% PCL $_{1250}$-HDI-DTH) appeared as the most uniform of the five samples with long, homogenous fibers, minor fiber fusing, minimal branching, no observed node formation, and a marked absence of the web-like secondary fibers present in Blends 2, 3, and 4 (Figure 6(e)).

3.5. Fiber Diameter. Table 1 lists the results of the fiber diameter and distribution analysis performed on the scaffolds of five blends. The full distribution is presented in
TABLE 2: Equivalent pore size of electrospun scaffolds (mean \pm standard deviation).

\begin{tabular}{lc}
\hline & Equivalent Diameter $(\mu \mathrm{m})$ \\
\hline Blend 1 & $4.9 \pm 1.7$ \\
Blend 2 & $10.4 \pm 2.8$ \\
Blend 3 & $8.2 \pm 1.2$ \\
Blend 4 & $9.8 \pm 2.3$ \\
Blend 5 & $9.8 \pm 2.2$ \\
\hline
\end{tabular}

Figure 7. Generally, the fiber diameter was between $1.2 \mu \mathrm{m}$ and $2.2 \mu \mathrm{m}$ across all blends. Blend $1,100 \% \mathrm{PEG}_{1000}-\mathrm{HDI}-$ DTH, possessed the statistically significant smallest mean diameter at $1193 \pm 227 \mathrm{~nm}$ while Blend $2\left(67 \% \mathrm{PEG}_{1000^{-}}\right.$ HDI-DTH, 33\% PCL $_{1250}$-HDI-DTH) possessed the largest at $2205 \pm 940 \mathrm{~nm}$. The mean fiber diameters of Blends 2, 3, 4, and 5 were not statistically different from one another. Fiber diameters for pure polyurethanes (Blends 1 and 5) appeared more uniform compared to those for the mixed polymers, as evidenced by the smaller standard deviations (Table 1). Due to the differing electrohydrodynamic behavior of the individual, chemically different polymers, the uniformly blended or pure solutions may allow for more regular spinning. The fiber diameters obtained in our work were well within the accepted general range of what can be considered for a well-formed tissue engineering scaffold.

Mean diameter measurement alone is insufficient to judge the quality of a fiber scaffold. A diameter distribution is necessary to provide more insight into overall properties of the electrospun fiber mat. A Gaussian distribution has been shown as a desirable trait for a tissue engineering scaffold [6], and thus it will be the approximate standard by which to judge the qualitative properties of the distribution. From Figure 7 , it can be seen that the fiber diameter distributions for Blends 1 and 2 fit the rough shape of a bell curve, that is, the mean, mode, and median occur near the same value. Blends 3 and 4 seem to be skewed toward the lower diameters. Blend 5 appears to possess minor bimodal behavior. Given the standard deviation, it is reasonable to believe that the $2200 \mathrm{~nm}$ bin may be anomalous for the chosen samples.

3.6. Pore Size. In addition to the fiber diameter, it is necessary for a tissue engineering scaffold to also mimic the pore size of the native ECM in order to facilitate cellular growth and proliferation [19]. The average diameter of human cells differs greatly throughout the body and thus it is necessary to match the pore size of a tissue engineering scaffold to the desired tissue/cell type [6].

Table 2 lists the average pore sizes of the blended Ltyrosine polyurethane scaffolds produced in this work. Blend 1 possessed the smallest equivalent diameter at $4.9 \pm 1.7 \mu \mathrm{m}$. Pore size of Blend 1 was also statistically smaller than that of the other four blends which averaged $9.6 \mu \mathrm{m}$. The equivalent pore diameters of Blends 2, 3, 4, and 5 were unable to be counted as statistically different from each other. From the table, it is clear that the scaffolds produced in our work 

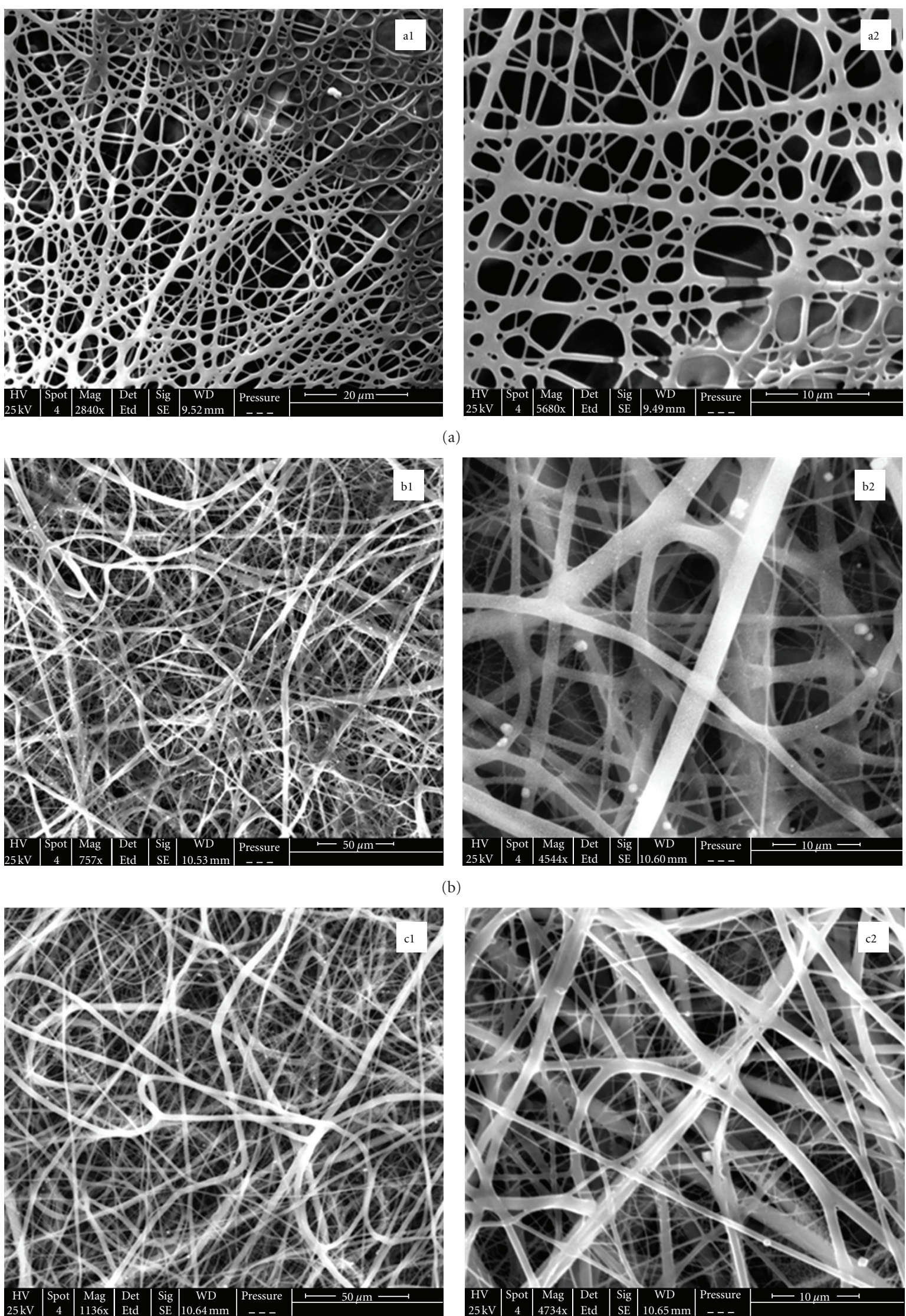

(c) 

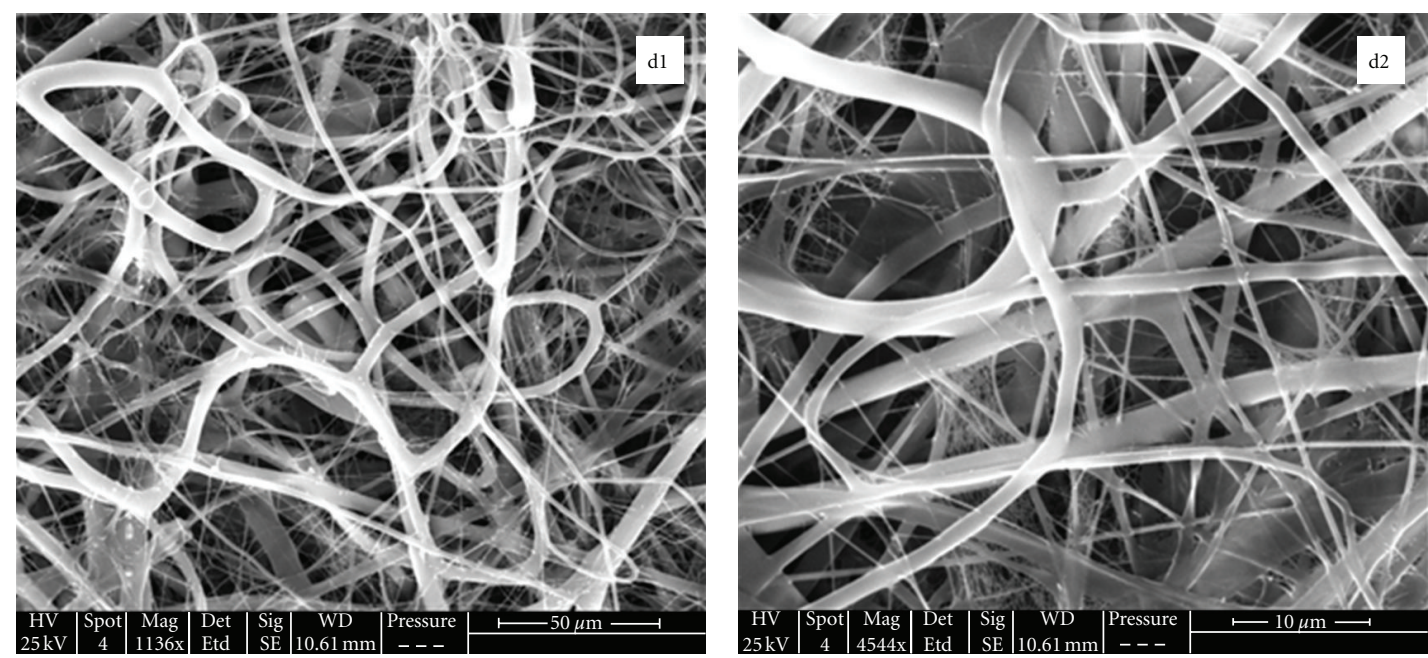

(d)
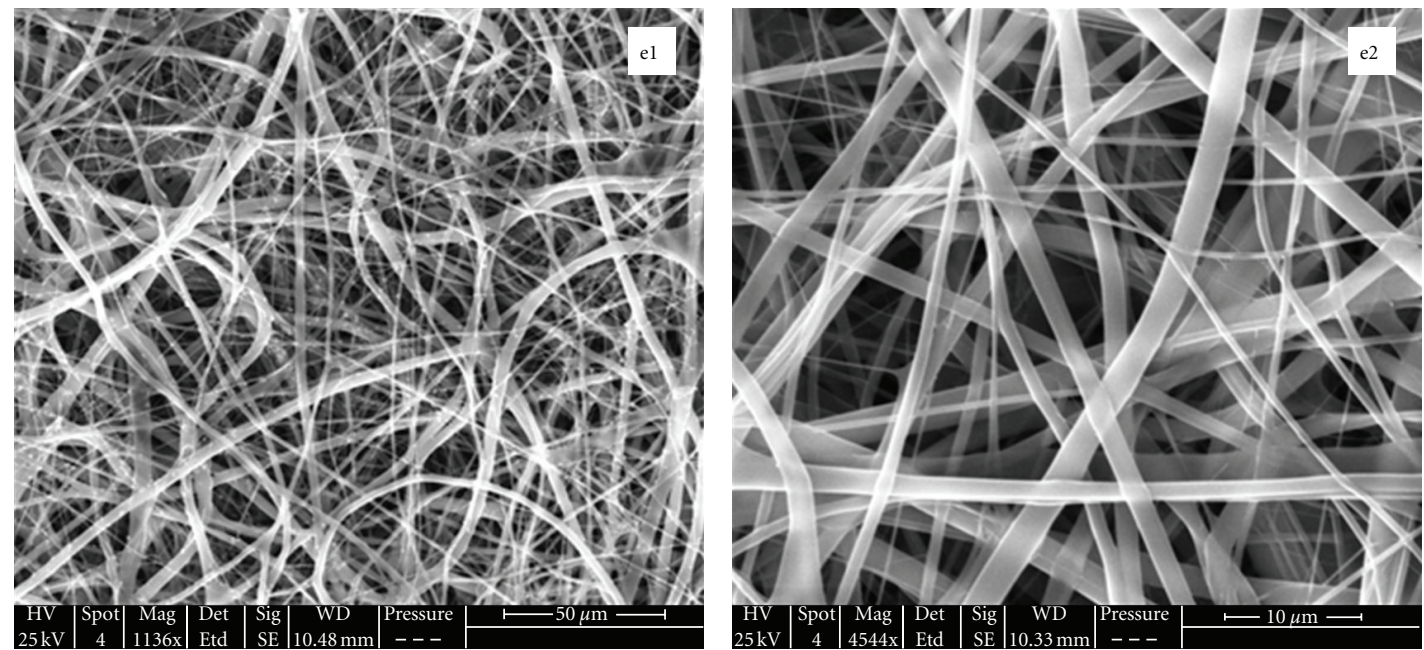

(e)

Figure 6: SEM images of electrospun scaffolds of Blend 1(a), Blend 2 (b), Blend 3 (c), Blend 4 (d), and Blend 5 (e).

possess equivalent pore sizes in the range of $5 \mu \mathrm{m}$ to $11 \mu \mathrm{m}$. The scaffolds should perform optimally in facilitating the growth and proliferation of human fibroblasts, for which the optimum range of pore size is $5-15 \mu \mathrm{m}$ [6]. Considering the thin nature of the electrospun mats, our scaffolds can be potentially used for the skin tissue engineering applications.

3.7. Hydrolytic Degradation. Two parallel hydrolytic degradation studies were performed: one on the electrospun scaffolds fabricated from each of the blended polyurethanes and the other on the thin films of each blended polymers. The parallel studies allow for accurate comparison between the structures (film and scaffold) without introducing additional variables from dissimilar stock polyurethanes. Specifically, the two studies allow observation of the effect of the increased surface area of the electrospun fibers relative to the solid thin film with respect to the hydrolytic degradation.

The results of the hydrolytic degradation performed on the fibrous electrospun scaffolds of the polymer blends are presented in Figure 8(a). The results clearly show that the polymer blends containing a higher percentage of $\mathrm{PEG}_{1000}$-HDI-DTH degraded to a greater extent than those containing more $\mathrm{PCL}_{1250}$-HDI-DTH over the same period of time. Overall, electrospun scaffolds of polyurethane blends degraded (i.e., lost mass), in order of decreasing $\mathrm{PEG}_{1000^{-}}$ HDI-DTH content, by $34.6 \pm 1.4 \%$ (Blend 1), $25.9 \pm 0.9 \%$ (Blend 2), $21.3 \pm 1.9 \%$ (Blend 3), $15.9 \pm 0.3 \%$ (Blend 4), and $9.2 \pm 0.3 \%$ (Blend 5), over a 60 -day hydrolytic degradation.

Solvent-cast thin films fabricated from the same blended L-tyrosine polyurethanes as the electrospun scaffolds were also subjected to hydrolytic degradation testing. The results of the experiments are shown in Figure 8(b). Much like the electrospun scaffolds, the blend films containing a higher percentage of $\mathrm{PEG}_{1000}-\mathrm{HDI}-\mathrm{DTH}$ degraded to a greater extent than their high $\mathrm{PCL}_{1250}$-HDI-DTH counterparts. This trend is consistent with the previous literature results $[14,16]$ and persists through all blend comparisons. The final values of mass loss for thin films after 35 days were $16.6 \pm 1.0 \%$ 


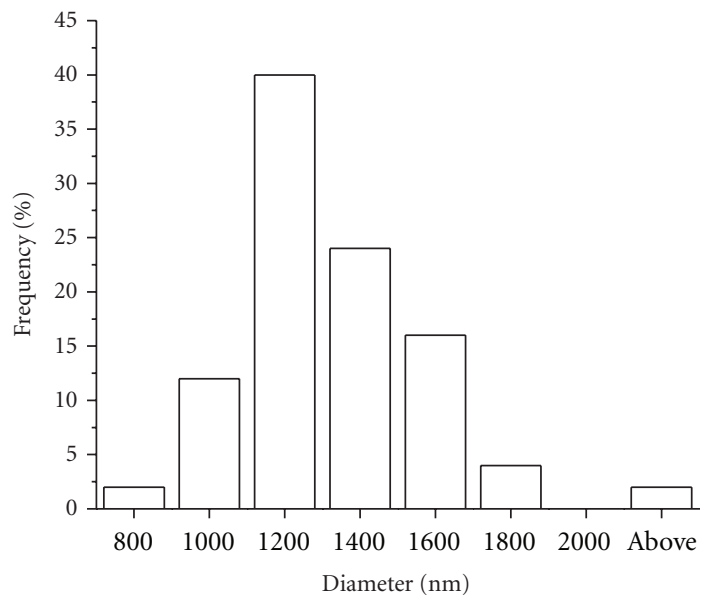

(a)

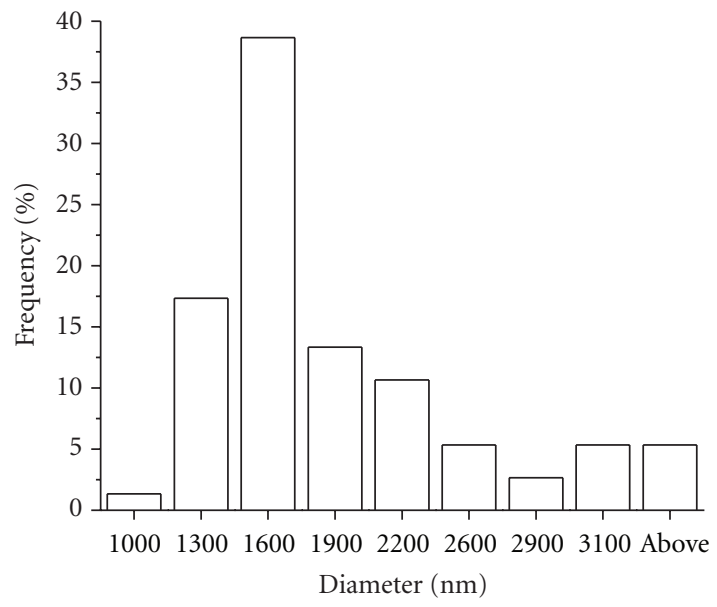

(c)

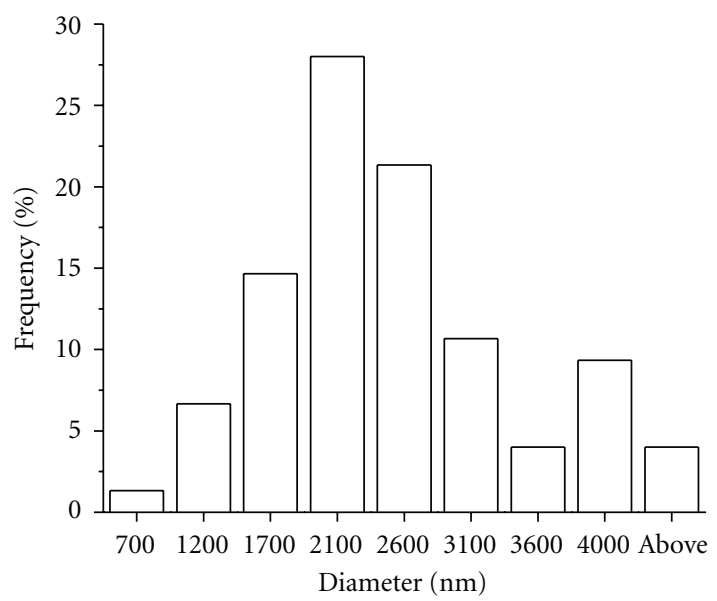

(b)

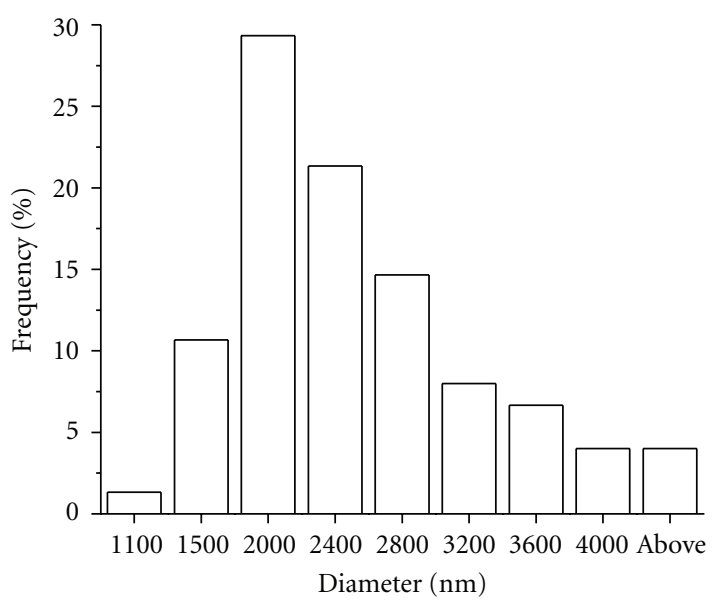

(d)

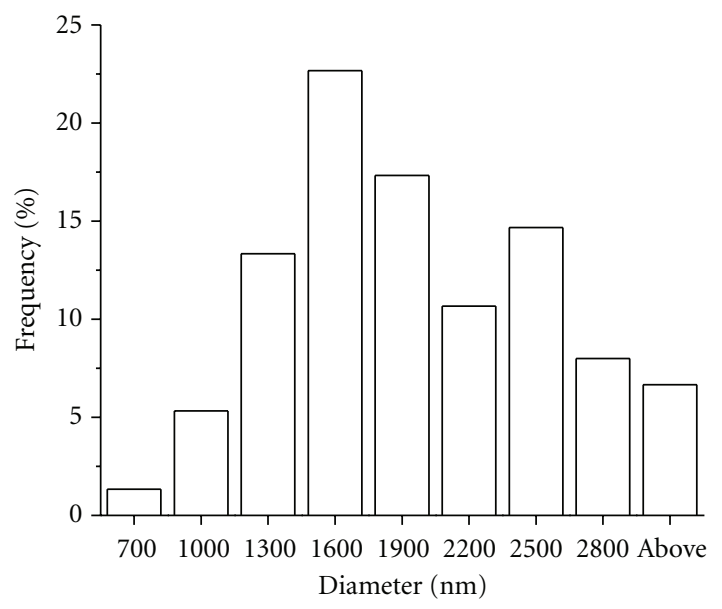

(e)

Figure 7: Fiber diameter distributions for each blended polyurethane scaffold: Blend 1 (a), Blend 2 (b), Blend 3 (c), Blend 4 (d), and Blend 5 (e). 


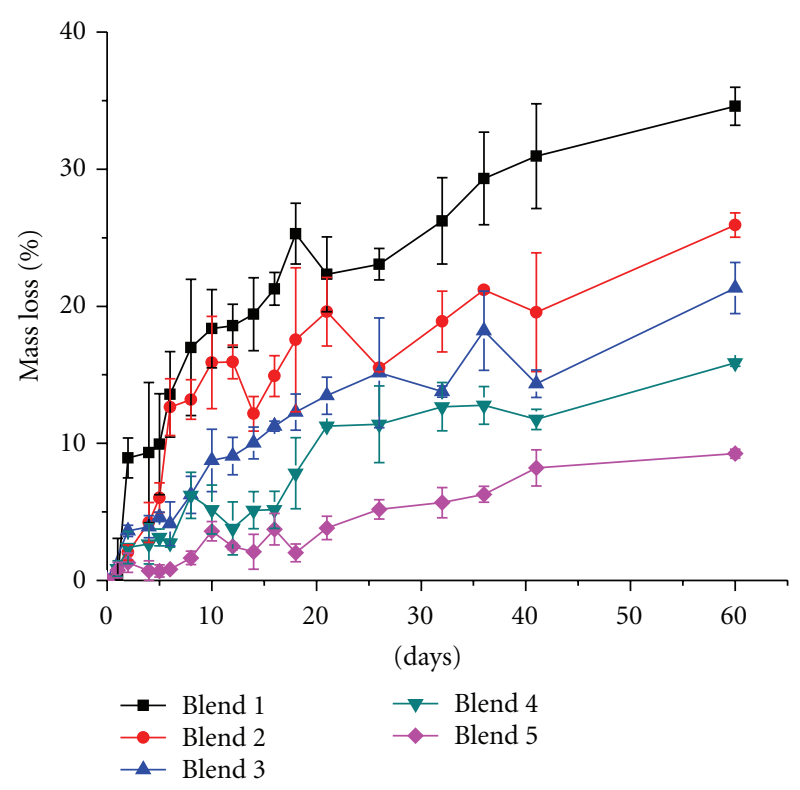

(a)

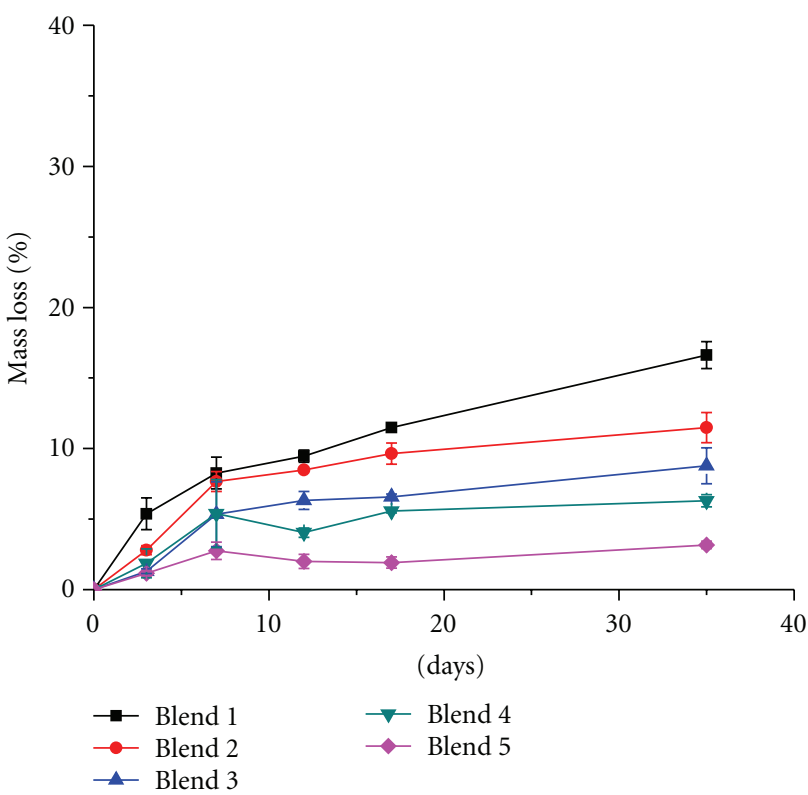

(b)

FIGURE 8: Mass loss profile of (a) electrospun scaffolds and (b) thin films from hydrolytic degradation. Data is presented as mean \pm standard error.

(Blend 1), $11.5 \pm 1.1 \%$ (Blend 2), $8.8 \pm 1.3 \%$ (Blend 3), $6.3 \pm 0.4 \%$ (Blend 4 ), and $3.2 \pm 0.3 \%$ (Blend 5 ).

It should be noted that, despite following the same pattern of PEG 1000 -HDI-DTH allowing for greater degradation, the electrospun scaffolds lost much more mass (as a percentage) than thin films over the same period of time. The difference in overall mass loss between the two forms (thin film and electrospun) at the 35-day mark can be seen in Table 3. Overall, the fiber form of the polymer blends lost $>76 \%$ more mass percentage than their thin
TABLE 3: Mass loss comparison between thin films and electrospun fibers after 35 days of hydrolytic degradation.

\begin{tabular}{lccc}
\hline & Thin film (\%) & Spun fiber (\%) & Increase over film (\%) \\
\hline Blend 1 & 16.6 & 29.3 & 76.3 \\
Blend 2 & 11.5 & 21.2 & 84.6 \\
Blend 3 & 8.8 & 18.2 & 107.5 \\
Blend 4 & 6.3 & 12.8 & 102.7 \\
Blend 5 & 3.2 & 6.3 & 98.1 \\
\hline
\end{tabular}

film counterparts. This is very likely due to the vastly increased surface area and porous structure generated by the electrospinning process through the formation of a multitude of nano- and microscale fibers composing the mat.

The value of these results is that they demonstrate that it is possible to fabricate tissue engineering scaffolds capable of degrading in a predictable way by simply altering the blend composition of two similar polymers. And often unspoken, yet majorly important characteristic of a biomaterial, especially a potentially implantable one, is how the material is handled by the body after the device has served its purpose. These results show that it is possible to modify L-tyrosine based polyurethanes in such a way that the degradation rate of the bulk material can be controlled, allowing yet another parameter for application-specific customization of a biomaterial. It should be noted that the degradation trials here were strictly hydrolytic. Other methods of decay, most notably enzymatic and oxidative means, will very likely affect and accelerate the process [20].

\section{Conclusions}

Two similar L-tyrosine-based polyurethanes were blended in the desired ratios, electrospun into morphologically acceptable fibrous scaffolds, characterized, and subjected to the hydrolytic degradation testing. Consistent with the hypothesis, the scaffolds containing a higher percentage of the less-resilient $\mathrm{PEG}_{1000}$-HDI-DTH degraded to a greater extent than those containing a lower percentage of the polymer (or a higher percentage of $\mathrm{PCL}_{1250}$-HDI-DTH). The trend of "increased PEG 1000 -HDI-DTH content yielding increased degree of degradation" held throughout all blends and configurations (electrospun scaffold and thin film). Furthermore, degradation of the electrospun scaffolds was faster than thin films with the same polymer compositions. While degradation results were notably different between blends, pore size and fiber diameter were not statistically distinct from one another across all blends. This suggests that the degradability of these scaffolds can be controlled without significantly affecting all morphological properties.

\section{Acknowledgments}

The authors thank Professor Stephanie T. Lopina (deceased) and Mr. Jui-Chen Yang for their assistance with the polymer synthesis and useful discussion. This work is supported by the Cleveland Clinic Foundation/Clinical Tissue Engineering 
Center (Ohio Department of Development TECH-09-006A), Firestone Research Initiative Fellowship, and the University of Akron Faculty Research Grant.

\section{References}

[1] S. L. M. Dahl, M. E. Vaughn, and L. E. Niklason, "An ultrastructural analysis of collagen in tissue engineered arteries," Annals of Biomedical Engineering, vol. 35, no. 10, pp. 17491755, 2007.

[2] E. Andreassen, E. L. Hinrichsen, K. Grøstad, O. J. Myhre, and M. D. Braathen, "Effects of the distributions of polypropylene fibre properties on the tensile response of fibre assemblies," Polymer, vol. 36, no. 6, pp. 1189-1198, 1995.

[3] R. Ogawa, J. Watanabe, and K. Ishihara, "Domain-controlled polymer alloy composed of segmented polyurethane and phospholipid polymer for biomedical applications," Science and Technology of Advanced Materials, vol. 4, no. 6, pp. 523530, 2003.

[4] U. Gbureck, O. Grolms, J. E. Barralet, L. M. Grover, and R. Thull, "Mechanical activation and cement formation of $\beta$ tricalcium phosphate," Biomaterials, vol. 24, no. 23, pp. 4123 4131, 2003.

[5] F. Tamimi, B. Kumarasami, C. Doillon et al., "Brushitecollagen composites for bone regeneration," Acta Biomaterialia, vol. 4, no. 5, pp. 1315-1321, 2008.

[6] S. Yang, K. F. Leong, Z. Du, and C. K. Chua, "The design of scaffolds for use in tissue engineering. Part I. Traditional factors," Tissue Engineering, vol. 7, no. 6, pp. 679-689, 2001.

[7] K. S. Midwood, L. V. Williams, and J. E. Schwarzbauer, "Tissue repair and the dynamics of the extracellular matrix," International Journal of Biochemistry and Cell Biology, vol. 36, no. 6, pp. 1031-1037, 2004.

[8] P. X. Ma and R. Zhang, "Synthetic nano-scale fibrous extracellular matrix," Journal of Biomedical Materials Research, vol. 46, no. 1, pp. 60-72, 1999.

[9] Y. Cao, T. I. Croll, A. J. O’Connor, G. W. Stevens, and J. J. Cooper-White, "Systematic selection of solvents for the fabrication of 3D combined macro- and microporous polymeric scaffolds for soft tissue engineering," Journal of Biomaterials Science, vol. 17, no. 4, pp. 369-402, 2006.

[10] Y. Barlow and J. Willoughby, "Pathophysiology of soft tissue repair," British Medical Bulletin, vol. 48, no. 3, pp. 698-711, 1992.

[11] J. Reignier and M. A. Huneault, "Preparation of interconnected poly(elunate-caprolactone) porous scaffolds by a combination of polymer and salt particulate leaching," Polymer, vol. 47, no. 13, pp. 4703-4717, 2006.

[12] M. E. Helgeson, K. N. Grammatikos, J. M. Deitzel, and N. J. Wagner, "Theory and kinematic measurements of the mechanics of stable electrospun polymer jets," Polymer, vol. 49, no. 12, pp. 2924-2936, 2008.

[13] A. Sen Gupta and S. T. Lopina, "L-tyrosine-based backbonemodified poly(amino acids)," Journal of Biomaterials Science, vol. 13, no. 10, pp. 1093-1104, 2002.

[14] D. Sarkar, J. C. Yang, and S. T. Lopina, "Structure-property relationship of L-tyrosine-based polyurethanes for biomaterial applications," Journal of Applied Polymer Science, vol. 108, no. 4, pp. 2345-2355, 2008.

[15] M. Shah, Fabrication and characterization of porous polyur-ethane scaffold for application in the field of tissue engineering, M.S. thesis, The University of Akron, Akron, Ohio, USA, 2008.
[16] P. N. Shah, R. L. Manthe, S. T. Lopina, and Y. H. Yun, "Electrospinning of 1-tyrosine polyurethanes for potential biomedical applications," Polymer, vol. 50, no. 10, pp. 22812289, 2009.

[17] W. D. Vilar, Chemistry and Technology of Polyurethanes, Vilar Poliuretanos, Rio de Janeiro, Brazil, 3rd edition, 2002.

[18] R. N. Pribble and T. S. Zwier, "Probing hydrogen bonding in benzene-(water)n clusters using resonant ion-dip IR spectroscopy," Faraday Discussions, vol. 97, pp. 229-241, 1994.

[19] D. W. Hutmacher, "Scaffold design and fabrication technologies for engineering tissues - state of the art and future perspectives," Journal of Biomaterials Science, vol. 12, no. 1, pp. 107-124, 2001.

[20] D. Sarkar and S. T. Lopina, "Oxidative and enzymatic degradations of 1-tyrosine based polyurethanes," Polymer Degradation and Stability, vol. 92, no. 11, pp. 1994-2004, 2007. 

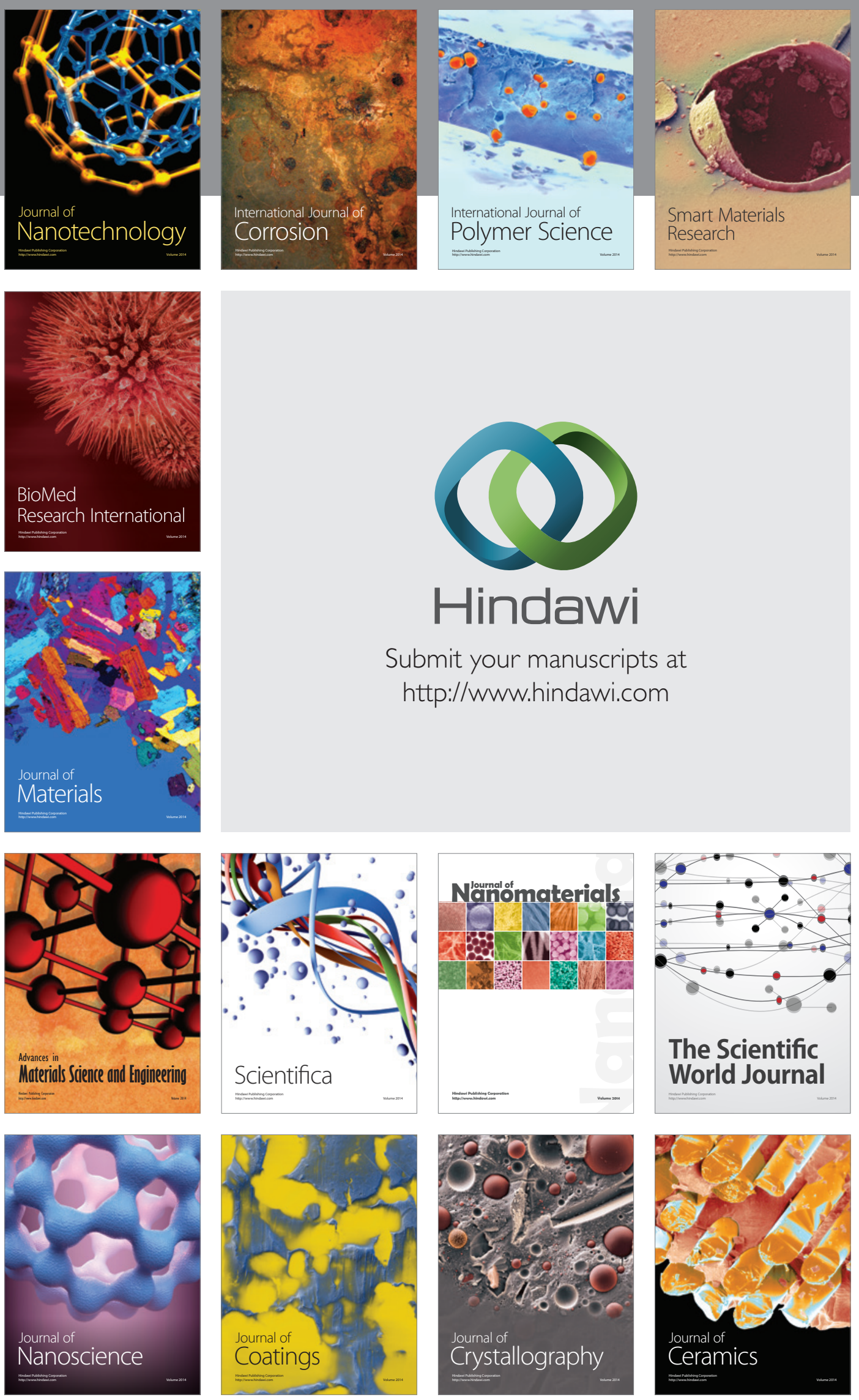

The Scientific World Journal

Submit your manuscripts at

http://www.hindawi.com

\section{World Journal}

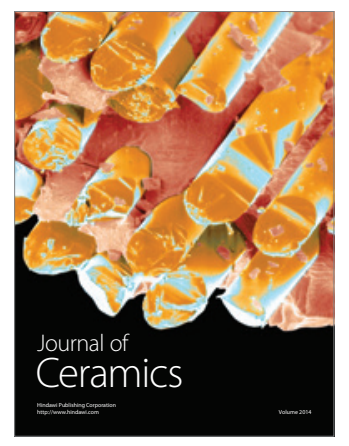

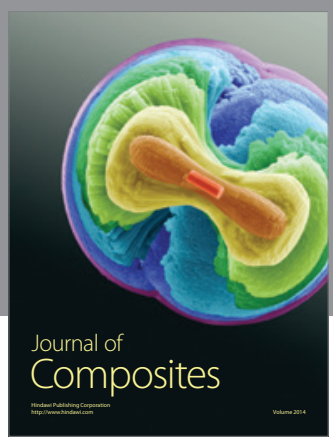
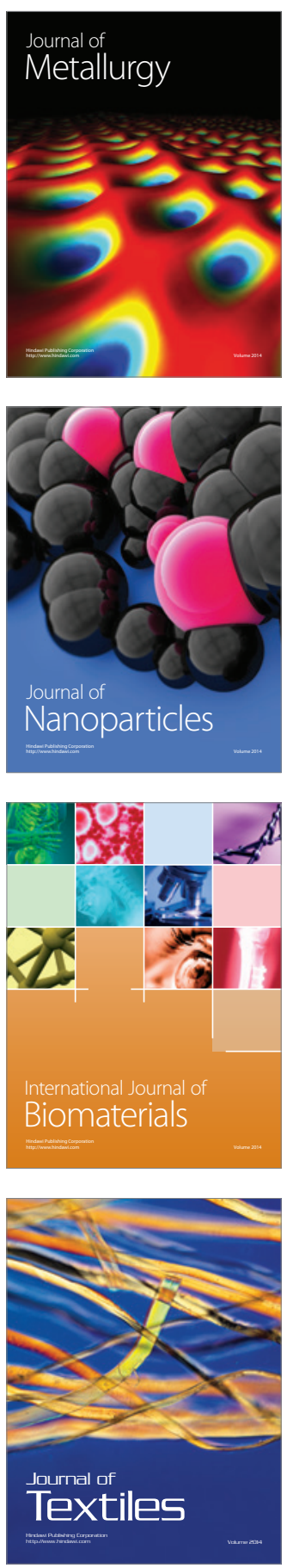